\section{Odds Ratio}

R.-D. Hilgers ${ }^{1}$, N. Heussen ${ }^{1}$ und S. Stanzel ${ }^{2}$

${ }^{1}$ Institut für Medizinische Statistik, Universitätsklinikum der RWTH Aachen, Aachen, Deutschland

${ }^{2}$ DKFZ Heidelberg, Heidelberg, Deutschland

Synonym(e) Chancenverhältnis; OR; Kreuzprodukt

Englischer Begriff odds ratio

Definition Das Odds Ratio beurteilt das Verhältnis zweier > Odds in Abhängigkeit einer Bedingung.

Beschreibung Das Odds Ratio (OR) ist ein Maß zur Beschreibung der Assoziation zwischen dem Vorliegen eines
Ereignisses und dem Vorhandensein einer Bedingung. Ein OR von 1 bedeutet, dass keine Assoziation vorliegt.

Der Begriff selbst hat eine enge Beziehung zum Vergleich von Wetten. Lässt sich für eine Wette die Gewinnchance auf einen Einsatz A mit 1:4 angeben, so lässt sich anhand des OR die relative Gewinnchance von A zu einem zweiten Einsatz B ausdrücken.

Die Berechnung von $95 \%$-Konfidenzintervallen (s. \& Konfidenzintervall) für das OR der $>$ Grundgesamtheit liefert eine Aussage darüber, ob das OR der Grundgesamtheit signifikant von 1 abweicht.

\section{Literatur}

Hilgers R-D, Bauer P, Scheiber V (2002) Einführung in die Medizinische Statistik. Springer, Berlin/Heidelberg/New York 